\title{
Big differences in primary care celiac disease serological markers request in Spain
}

\author{
Maria Salinas ${ }^{* 1,2}$, Maite López-Garrigós', Emilio Flores¹,3, Carlos Leiva-Salinas ${ }^{4}$ \\ On behalf of the Pilot Group of the Appropriate Utilization of Laboratory Tests \\ ${ }^{1}$ Clinical Laboratory, Hospital Universitario de San Juan, San Juan de Alicante, Spain \\ ${ }^{2}$ Department of Biochemistry and Molecular Pathology, Universidad Miguel Hernandez, Elche, Spain \\ ${ }^{3}$ Department of Clinical Medicine, Universidad Miguel Hernandez, Elche, Spain \\ ${ }^{4}$ Department of Radiology, University of Virginia, Charlottesville, Virginia, USA \\ *Corresponding author: salinas_mar@gva.es
}

\begin{abstract}
Introduction: Celiac disease (CD) prevalence is increasing but the disorder remains undiagnosed. The study compares $C D$ serology markers requested by General Practitioners (GPS) over time and geographical areas. The aim of the current research is to assess the inter-practice and temporal variability in the request of $C D$ serology markers by GPs in Spain, and the differences between regions.

Materials and methods: A cross-sectional study was conducted enrolling Spanish clinical laboratories. Primary care CD serology markers request in 2010, 2012 and 2014 from 15 autonomous communities (AACC), with more participants was reported. Test-utilization rates were calculated (tissue transglutaminase $\lg A$ antibodies (TTG-IgA) and deaminated peptide gliadine $\lg A$ antibodies (DGP-lgA) per 1000 inhabitants), and also the ratio of both tests request (DGP-IgA/tTG-IgA).

Results: The request of tTG-IgA per 1000 inhabitants increased significantly along years (from 3.99 to $5.90(P<0.001)$ ). The demand of DGP-IgA per 1000 inhabitants was maintained in 2010 and 2012 (0.68 and 0.6), and decreased in $2014(0.35)(P=0.927)$. DGP-IgA/tTG-IgA diminished over time (from 0.16 to $0.06(P=0.548))$, and in the 2014 edition, there was a significant regional difference, ranging from 0.01 to $0.57(P<0.001)$.

Conclusions: The variability in the request in $C D$ serology markers emphasizes the need of inter-regional cooperation to develop strategies to optimize the use of laboratory tests.
\end{abstract}

Key words: celiac disease; quality indicators; clinical laboratory services; benchmarking

\section{Introduction}

Celiac disease (CD) is an immune-mediated small intestinal enteropathy that is activated by exposure to dietary gluten in genetically predisposed individuals. Originally $C D$ was considered a rare malabsorption syndrome affecting mainly children, but nowadays it has been recognized as a common condition that may be diagnosed at any age (1). CD is characterized by the presence of a variety of gluten-dependent clinical manifestations, specific antibodies of $C D$, haplotypes HLA-DQ2 and/or HLA-DQ8 and enteropathy.
Traditionally patients presented with malabsorption but over time the proportion of newly diagnosed patients with malabsorptive symptoms have decreased and even asymptomatic or patients with variable non-gastrointestinal findings have increased (2). Patients with CD can present with a wide range of symptoms and signs, and are classically diagnosed through a positive serology and ulterior duodenal biopsy while consuming a gluten-containing diet. Due to the diversity of the clinical symptoms, presentation at any age and the increasing prevalence, $C D$ is more often diag- 
nosed in primary care by the General Practitioners (GPs) who serve as the patients' first point of contact (3).

The disease prevalence varies considerably, is increasing worldwide and many patients with $C D$ still remain undiagnosed (4). In fact it is estimated that in 2020 there will be 5 million cases of CD cases just in the Mediterranean area (5).

Specific antibodies of $C D$ are tissue transglutaminase $\lg A$ antibodies (tTG- $\lg A$ ), endomysial $\lg A$ antibodies (EmA), and the deamidated gliadin peptide $\lg A$ antibodies (DGP-IgA).

Since publication of the European Society of Paediatric Gastroenterology and Nutrition (ESPGHAN) guidelines in year 2012, tTG-IgA antibody is the preferred single test for detection of CD (6). Tests that measure DGP-IgA may be used as additional tests in patients who are negative for other CDspecific antibodies but in whom clinical symptoms raise a strong suspicion of $C D$, especially if they are younger than 2 years. In subjects with humoral $\lg A$ deficiency, at least 1 additional test measuring IgG class CD-specific antibodies should be done (6).

The diversity of clinical symptoms and the increasing prevalence emphasize the need for strategies for the optimal detection of patients. A first step would be to study how appropriately CD serology is requested in primary care. A previous study has shown a higher variability in the rarely requested tests in primary care, in a population covering around $38 \%$ of the Spanish population (7).

We hypothesized that there is a high variability in the use of serological markers of CD in Primary Care. Thus, the aim of this study is to assess the inter-practice and temporal variability in the requests of serological markers of CD from primary care as well as to assess the differences between regions in Spain.

\section{Materials and methods}

\section{Study Design}

A cross-sectional study was conducted at the national level, enrolling clinical laboratories belonging to the Autonomous Communities (AACC) of
Spain, all of which operate under the Spanish $\mathrm{Na}$ tional Health Service, which is responsible for the majority of the national population. All applicable residents have free access to their primary care physician and to the hospital.

The study was designed and conducted at the University Hospital of San Juan de Alicante, where a questionnaire (Supplementary material) was developed and used to collect different variables from Spanish laboratories: number of tests performed from the Laboratory Information Systems Patient's databases and organizational data in three different years. In 2010 a call for data was posted on the Redconlab website, and also via email in the Redconlab 2012 study. In the edition of 2014, the dissemination of the questionnaire was also addressed through a Linkedln (https:// www.linkedin.com/in/redconlab-grupoa5663bb7) group. In the three different years, or editions, participation in the REDCONLAB study was voluntary. In all, 37, 76 and 110 laboratories, on a voluntary basis, participated in the 2010, 2012 and 2014 editions.

\section{Methods}

Numbers of tTG-IgA and DGP-IgA, requested by all the GPs for the years 2010, 2012 and 2014 from laboratories at different health departments (HD) across Spain were reported in the three studies.

Test-utilization rates were calculated. Rates were expressed as tests (tTG-IgA and DGP-IgA) per 1000 inhabitants, and also through the ratio of both tests requests (DGP-IgA /tTG-lgA). The three editions results were compared.

In the 2014 edition, laboratories were grouped in the different AACC, when more than 4 participants, and a group joining the results of the rest. AACC were codified by numbers due to confidentiality, and DGP-lgA/tTG-lgA ratio was calculated.

\section{Statistical analysis}

The analysis of the distribution of the indicators was conducted by Kolmogorov-Smirnov test. The numerical data (tTG-IgA and DGP-IgA per 1000 inhabitants, and the ratio of both tests requests 
(DGP-lgA /tTG-lgA), are presented as median (interquartile range). The differences in the indicators between years and AACC were calculated using the Kruskal-Wallis test analysis. A two-sided $\mathrm{P} \leq$ 0.001 rule was utilized as the criterion for rejecting the null hypothesis of no difference. All statistical analyses were carried out with SPSS version 22.0 (SPSS, Chicago, IL, USA).

\section{Results}

Table 1 shows the data of all participants and also the descriptive analysis of the three indicators in the three editions.

The request of tTG-IgA expressed per 1000 inhabitants increased along years from 3.99 to $5.90(P<$ 0.001). On the other hand, the demand of DGP-IgA was maintained in the first two editions, and decreased in the third, although this difference was not statistically significant. The DGP-lgA/tTG-lgA ratio mildly diminished over time.

The 2014 edition joined 10 AACCS with more than 4 participants (in alphabetical order: Andalucía, Canarias, Castilla La Mancha, Castilla Leon, Extremadura, Galicia, Madrid, Murcia, País Vasco and Valencia). An eleventh group was created with those AACC that did not reach the 4 participants (in alphabetical order: Aragon, Asturias, Baleares, Cantabria and Cataluña).
Figure 1 shows graphically the DGP-lgA/tTG-IgA indicator results for year 2014 in the different AACC; there was a significant difference between regions, ranging from 0.01 to $0.57(P<0.001)$.

\section{Discussion}

As expected, the primary care anti-tTG request increased along years and the ratio DGP-IgA/tTG$\lg A$ decreased. No decrease was observed in the request of DGP-IgA in the first two editions, despite its request should have been limited to patients less than two years of age. There were big regional differences in the DGP-IgA/anti-tTG-IgA indicator result.

Although participants did not state if they implemented guidelines or not, the increment in tTG$\lg \mathrm{A}$ request along years was according to the guidelines for the diagnosis of CD. It took, however, more time to observe a subsequent decreased in the requests in DGP-IgA. It is true that the illness is more frequently detected in children, but the rates of DGP-IgA request seemed excessive at least in the first two editions, taking into account that only $2.85 \%$ of the Spanish population is younger than 2 years (8). Conversely, 6 AACCs showed very low results in that indicator, suggesting an earlier adoption of the new guidelines. However, the differences between regions of DGP-IgA/anti-tTG-

TABLE 1. Data and descriptive analysis of indicators in the three REDCONLAB editions

\begin{tabular}{|c|c|c|c|c|}
\hline & \multicolumn{3}{|c|}{ REDCONLAB EDITION } & \multirow{7}{*}{ P value } \\
\hline & 2010 & 2012 & 2014 & \\
\hline Centers, $\mathbf{N}$ & 37 & 76 & 110 & \\
\hline AACC, $\mathbf{N}$ & 8 & 13 & 15 & \\
\hline Inhabitants attended, N & $8,130,334$ & $17,679,195$ & $27,434,262$ & \\
\hline Total DGP-IgA, N & 8530 & 34,371 & 38,178 & \\
\hline Total tTG-IgA, N & 34,996 & 86,962 & 169,097 & \\
\hline DGP-IgA/1000 inhabitants & $0.68(2.08)$ & $0.60(2.51)$ & $0.35(2.03)$ & 0.927 \\
\hline tTg-IgA/1000 inhabitants & $3.99(3.20)$ & $4.37(3.45)$ & $5.90(4.30)$ & $<0.001$ \\
\hline DGP-IgA/tTG-IgA ratio & $0.16(0.76)$ & $0.12(0.76)$ & $0.06(0.46)$ & 0.548 \\
\hline
\end{tabular}

AACC - Autonomous communities. tTG-IgA - tissue transglutaminase IgA antibodies. DGP-IgA - deamidated gliadin peptide IgA antibodies. Results are presented as median (interquartile range). The differences in the indicators between years were calculated using the Kruskal-Wallis test analysis. $\mathrm{P} \leq 0.001$ was considered statistically significant. 


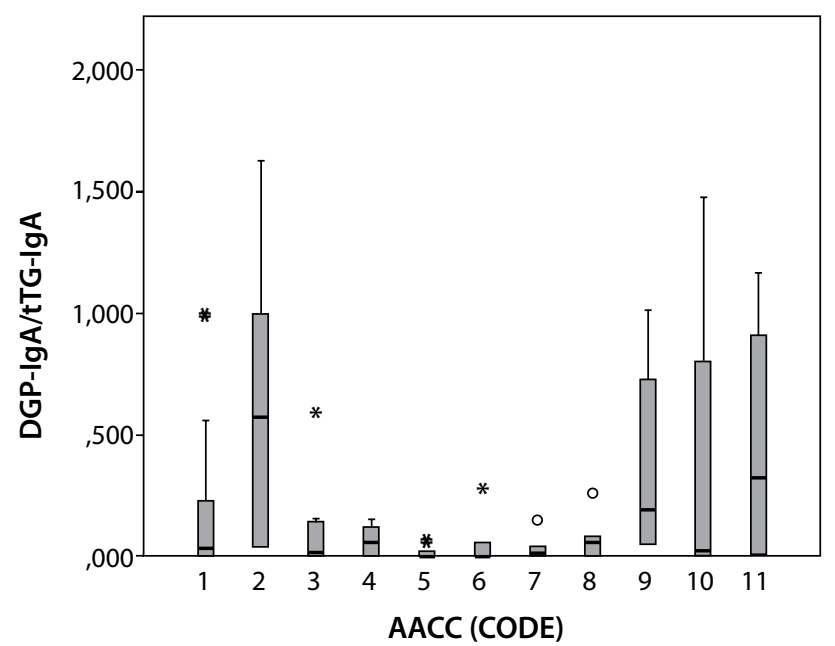

\begin{tabular}{|c|c|c|c|c|c|c|c|c|c|c|c|c|}
\hline \multicolumn{13}{|c|}{ AACC code } \\
\hline & 1 & 2 & 3 & 4 & 5 & 6 & 7 & 8 & 9 & 10 & 11 & \multirow[t]{2}{*}{$P$ value } \\
\hline Centers (N) & 20 & 16 & 10 & 11 & 12 & 5 & 5 & 6 & 6 & 5 & 14 & \\
\hline Median & 0.05 & 0.57 & 0.01 & 0.05 & 0.00 & 0.00 & 0.01 & 0.06 & 0.19 & 0.02 & 0.32 & \multirow{2}{*}{$<0.001$} \\
\hline IQR & 0.56 & 0.96 & 0.15 & 0.12 & 0.01 & 0.05 & 0.04 & 0.08 & 0.68 & 0.78 & 0.9 & \\
\hline
\end{tabular}

IQR - interquartile range. AACC - Autonomous communities. The differences in the indicator between years was calculated using the Kruskal-Wallis test analysis. $\mathrm{P} \leq 0.001$ was considered statistically significant.

Figure 1. Bloxpot of ratio of tests requests (DGP-lgA /tTG-IgA) in different AACC.

tTG-IgA - tissue transglutaminase IgA antibodies. DGP-IgA - deamidated gliadin peptide IgA antibodies. AACC - Autonomous communities. ${ }^{\circ}$ - outlier. ${ }^{*}$ - extreme value.

IgA indicator results suggest that is probably due to different requesting customs in the different AACCs, or maybe different timing in the implementation of new clinical guidelines.

Our research is the first to study how CD serological tests are used over time in $C D$, a disorder prone to active case-finding strategy in primary care, to effectively improve its diagnostic rate (9).

Our results show that there is a need for a faster dissemination of scientific evidence, especially when it deals with a common and relevant disorder such as CD. In fact, an early diagnosis is clinically relevant, as possible evolution of undiagnosed cases include non-Hodgkin's Lymphoma, carcinomas or refractory CD. Our study also shows that the establishment of strategies is crucial to reach a homogeneous, appropriate and efficient request of diagnostic tests. There are interventions, that once designed through the application of scientific evidence and consensus with GPs, could be maintained over time, such as computeraided algorithms that could substitute inappropriate tests for meaningful ones, as, in this case DGP$\lg A$ for $\mathrm{tTG}-\lg A$ when the patient is older than 2 years (10).

The main limitation of the study is that the differences in test requesting patterns, could be explained by case mix variations or groups of patients requiring similar tests, procedures, and resources, and also the number of children and adults among habitants attended in the different HD or AACC.

The variability in the request in $C D$ serology markers, the delay in the demand of appropriate tests and the significant difference between AACC emphasize the need to improve communication and to establish interventions to enhance the appropriate use of laboratory tests to diagnose CD. Inter- 
departmental and inter-regional cooperation would be crucial to develop strategies in order to optimize the use of laboratory tests.

\section{Acknowledgements}

Members of the REDCONLAB working group are the following: Vidal Perez Valero (Hospital Regional Universitario de Malaga, Hospital Universitario Virgen de la Victoria); Félix Gascón (Hospital Valle de los Pedroches, Malaga, Pozoblanco); Isidoro Herrera Contreras (Complejo Hospitalario de Jaen); Maria Angeles Bailen Garcia (Hospital Universitario Puerta del Mar de Cadiz); Cristobal Avivar Oyonarte (Hospital de Poniente, El Ejido); Esther Roldán Fontana (A.G.S. de Osuna); Fernando Rodriguez Cantalejo (Hospital Universitario Reina Sofia de Cordoba); Jose Angel Noval Padillo (Hospital Universitario Virgen del Rocio); M Angela Gonzalez García (AGS Norte de Cadiz); Ignacio Vazquez Rico (Hospital Juan Ramon Jimenez de Huelva); Cristina Santos (Hospital Rio Tinto); Angeles Giménez Marín (Hospital de Antequera); Maria del Señor López Vélez (Hospital San Cecilio de Granada), Jose Vicente Garcia Larios (Hospital Virgen de las Nieves, Granada); Federico Navajas Luque (AGS Este de Málaga-Axarquía); Amado Tapia (Hospital de Barbastro); Maria Esther Sole LLop (Hospital de Alcañiz); Juan José Puente (Hospital Clínico Universitario Lozano Blesa); Patricia Esteve (Hospital Ernest Lluch); Maria Teresa Avello Lopez (Hospital San Agustín-Aviles, Hospital Valle del Nalon); Emilia Moreno Noguero (Hospital Can Mlsses); Ana Maria Follana Vazquez (Hospital Mateu Orfila); Jose Luis Ribes Valles (Hospital de Manacor); Ma Luisa Fernández de Lis Alonso (Area de Salud de Fuerteventura); Cecilia Martin (Hospital Nuestra Señora de la Candelaria, Tenerife); Leopoldo Martin Martin (Hospital General de la Palma); Miguel Angel Pico Picos (Hospital Universitario de Canarias); Casimira Dominguez Cabrera (Hospital Universitario de Gran Canaria Dr Negrín); Marta Riaño Ruiz (Hospital Insular de Gran Canaria); Juan Ignacio Molinos (Hospital Sierrallana de Torrelavega); Luis Fernando Colomo (Hospital de Laredo); Marcos Lopez Hoyos (Hospital Universitario Marques de Valdecilla); Enrique Prada de Medio (Hospital Virgen de la Luz de Cuenca); Pilar Garcia Chico
(Hospital General Universitario de Ciudad Real); Simon Gomez-Biedma (Hospital General de Almansa); Vicente Granizo Dominguez (Hospital Universitario de Guadalajara); Guadalupe Ruiz (Complejo Hospitalario de Toledo); Laura Navarro (Complejo Hospitalario Universitario de Albacete); Fidel Velasco Pena (Hospital Virgen de Altagracia, Manzanares); Carolina Andrés Fernandez (Hospital General de Villarobledo); Joaquín Domínguez Martinez y Oscar Herráez Carrera; Ma Carmen Lorenzo Lozano (Hospital de Puertollano); Maria Teresa Gil (Hospital Nuestra Señora del Prado Talavera de la Reina); Ma Angeles Rodriguez Rodriguez (Complejo Asistencial Universitario de Palencia (Hospital Rio Carrion); M. Victoria Poncela Garcia (Hospital Universitario de Burgos); Luis Rabadan (Complejo Asistencial de Soria); Vicente Villamandos (Hospital Santos Reyes, Aranda del Duero); Nuria Fernandez Garcia (Hospital Universitario Rio Hortega-Valladolid); Jose Miguel Gonzalez Redondo (Hospital Santiago Apostol de Miranda de Ebro); Cesareo Garcia (Hospital Universitario de Salamanca); Luis Garcia Menendez (Hospital El Bierzo); Pilar Alvarez Sastre (Complejo Asistencial de Zamora); Ovidio Gomez (Hospital Cínico Universitario de Valladolid); Mabel LLovet (Hospital Universitario Verge de la Cinta (Tortosa)); Nuria Serrat (Hospital Joan XXIII de Tarragona); Ma José Baz (Hospital de Llerena, Badajoz); Maria Jose Zaro (Hospital Don Benito-Villanueva); M Carmen Plata (Hospital Campo Arañuelo, Navalmoral de la Mata); Pura Garcia Yun (Área de Salud de Badajoz (Hospital Infanta Cristina, Hospital Perpetuo Socorro y Hospital Materno Infantil); Milagrosa Macías Sánchez (Area de Salud de Caceres (Complejo Hospitalario San Pedro de Alcantara); Javier Martin (Hospital Virgen del Puerto de Plasencia); Lola Máiz Suarez (Hospital Lucus Augusti, Lugo); Berta Gonzalez Ponce (Hospital Da Costa, Burela); Aida Perez Fuertes (Hospital Arquitecto Marcide, El Ferrol); M. Amalia Andrade Olivie (Hospital Xeral-Cies, CHU Vigo); Pastora Rodriguez (Hospital Universitario de A Coruña); M. Mercedes Herranz Puebla (Hospital General Universitario Gregorio Marañon); Antonio Buño Soto (Hospital Universitario La Paz, Madrid); Fernando Cava (BR Salud); Tomas Pascual (Hospital Universitario Raquel Guillén Santos de Getafe); Carmen Hernan- 
do de Larramendi (Hospital Severo Ochoa de Leganes); Raquel Blázquez Sánchez (Hospital de Mostoles); Pilar Díaz (Hospital 12 de Octubre, Madrid); Ana Díaz (Hospital Universitario de La Princesa); Marta Garcia Collia (Hospital Ramon y Cajal, Madrid); Maria Angeles Cuadrado Cenzual (Hospital Clinico San Carlos); Santiago Prieto (Hospital Universitario de Fuenlabrada); María Menchero del Carmen Gallego Ramírez (Hospital Rafael Mendez, Lorca); Jose Luis Quilez Fernandez (Hospital Universitario Reina Sofia de Murcia); Maria Dolores Albaladejo (Hospital Santa Lucia, Cartagena); Maria Luisa Lopez Yepes (Hospital Virgen del Castillo de Yecla); Alfonso Pérez Martínez (Hospital Morales Meseguer); Antonio López Urrutia (Hospital de Cruces, Bilbao); Adolfo Garrido Chércoles (Hospital Universitario de Donostia); Carmen Mar Medina (Hospital Galdakao-Usonsolo); M Carmen Zugaza (Unidad de Gestion Clinica de Alava); Patxi Aguayo (Hospital Universitario de Basurto); Silvia Pesudo (Hospital La Plana); Carmen Vinuesa (Hospital de Vinaros); Julian Díaz (Hospital Francesc de Borja,
Gandia); Marisa Graells (Hospital General Universitario de Alicante); Diego Benitez Benitez (Hospital de Orihuela); Arturo Carratala (Hospital Clinico Universitario de Valencia); Consuelo Tormo (Hospital General de Elche); Francisco Miralles (Hospital Lluis Alcanyis, Xativa); Amparo Miralles (Hospital de Sagunto); Jose Luis Barberà (Hospital de Manises); Juan Molina (Hospital Comarcal de La Marina, Villajoyosa); Martin Yago (Hospital de Requena); Mario Ortuño (Hospital Universitario de la Ribera (Alzira)); Maria Jose Martinez Llopis (Hospital de Denia); Nuria Estañ (Hospital Dr. Peset); Ricardo Molina (Hospital Virgen de los Lirios, Alcoy); Juan Antonio Ferrero (Hospital General de Castellon); Begoña Laiz Marro (Hospital Universitario y Politecnico La Fe de Valencia); Goitzane Marcaida (Consorcio Hospital General Universitario de Valencia).

\section{Potential conflict of interest}

None declared.

\section{References}

1. Rampertab SD, Pooran N, Brar P, Singh P, Green PH. Trends in the presentation of celiac disease. Am J Med 2006;119:355. https://doi.org/10.1016/j.amjmed.2005.08.044.

2. Lindfors $K$, Koskinen $O$, Kaukinen K. An update on the diagnostics of celiac disease. Int Rev Immunol 2011;30:185-96. https://doi.org/10.3109/08830185.2011.595854.

3. Husby S, Koletzko S, Korponay-Szabo I, Mearin L, Philips A, Shamir R, et al. ESPGHAN guidelines for the diagnosis for celiac disease in children and adolescents. An evidence-based approach. J Pediatr Gastroenterol Nutr 2012;54:136-60. https://doi.org/10.1097/MPG.0b013e31821a23d0.

4. Murray JA, Van Dyke C, Plevak MF, Dierkhising RA, Zinsmeister AR, Melton LJ 3rd. Trends in the identification and clinical features of celiac disease in a North American community, 1950-2001. Clin Gastroenterol Hepatol 2003;1:19-27. https://doi.org/10.1053/jcgh.2003.50004.

5. Greco L, Timpone L, Abkari A, Abu-Zekry M, Attard T, Bouguerrà $F$, et al. Burden of celiac disease in the Mediterranean area. World J Gastroenterol 2011;17:4971-8. https:// doi.org/10.3748/wjg.v17.i45.4971.

6. Rashid M, Lee J. Serologic testing in celiac disease: Practical guide for clinicians. Can Fam Physician 2016;62:38-43.

7. Salinas M, López-Garrigós M, Flores E, Uris J, Leiva-Salinas C; Pilot Group of the Appropriate Utilization of Laboratory Tests. Larger differences in utilization of rarely requested tests in primary care in Spain. Biochem Med (Zagreb) 2015;25:410-5. https://doi.org/10.11613/BM.2015.041.

8. Instituto Nacional de Estadística. Available at: http://www. ine.es/. Accessed June 29th 2016.

9. Catassi C, Kryszak D, Louis-Jacques O, Duerksen DR, Hill I, Crowe SE, et al. Detection of celiac disease in primary care: a multicenter case-finding study in North America. Am J Gastroenterol 2007;102:1454-60. https://doi.org/10.1111/ j.1572-0241.2007.01173.x.

10. Salinas $M$, López-Garrigós $M$, Asencio A, Leiva-Salinas $M$, Lugo J, Leiva-Salinas C. Laboratory utilization improvement through a computer-aided algorithm developed with general practitioners. Clin Chem Lab Med 2015;53:1391-7. https://doi.org/10.1515/cclm-2014-0762. 